\title{
On The Road To A Neuroprosthetic Hand: A Novel Hand Grasp Orthosis Based on Functional Electrical Stimulation
}

\author{
Robert Leeb, Miguel Gubler, Michele Tavella, Heather Miller and José del. R. Millán
}

\begin{abstract}
To patients who have lost the functionality of their hands as a result of a severe spinal cord injury or brain stroke, the development of new techniques for grasping is indispensable for reintegration and independency in daily life. Functional Electrical Stimulation (FES) of residual muscles can reproduce the most dominant grasping tasks and can be initialized by brain signals. However, due to the very complex hand anatomy and current limitations in FES-technology with surface electrodes, these grasp patterns cannot be smoothly executed. In this paper, we present an adaptable passive hand orthosis which is capable of producing natural and smooth movements when coupled with FES. It evenly synchronizes the grasping movements and applied forces on all fingers, allowing for naturalistic gestures and functional grasps of everyday objects. The orthosis is also equipped with a lock, which allows it to remain in the desired position without the need for long-term stimulation. Furthermore, we quantify improvements offered by the orthosis compare them with natural grasps on healthy subjects.
\end{abstract}

Index Terms-Orthosis, FES, BCI, EEG, neuroprosthetics, grasping, rehabilitation

\section{INTRODUCTION}

If in our daily life we intend to grasp an object, we do not think how to reach the object, how to pre-shape our hand, or how much force we need to apply to safely hold it. Everything is controlled by our nervous system including voluntary intentions and complicated visual, proprioceptive and tactile feedback. But how can people who have lost the use of their hands due to a spinal cord injury or brain stroke regain the ability to make natural grasps like their healthy counterparts? Is it possible for them to regain the ability to grasp with their fully paralyzed hand by controlling assistive devices using their thoughts alone?

For amputees, many fully actuated prostheses can replace the functionalities of the lost limb. Recent work has even provided devices that are neurologically controlled, e.g. by targeted muscle reinnervation or electromyography of peripheral nerves. But for patients who have not lost limbs and are instead paralyzed, there has unfortunately been only

This research is supported by the European ICT Programme Project FP7-224631, TOBI: Tools for Brain-Computer Interaction. This paper only reflects the authors' views and funding agencies are not liable for any use that may be made of the information contained herein.

R. Leeb, M. Tavella, H.Miller and J.d.R. Millán are with the Chair on Non-Invasive Brain-Machine Interface, Center for Neuroprosthetics, School of Engineering, Ecole Polytechnique Fédérale de Lausanne, CH-1015 Lausanne, Switzerland, \{robert.leeb, michele.tavella, heather.miller, jose.millan\} depfl.ch

M. Gubler is with the Sensory-Motor System Lab, Swiss Federal Institute of Technology Zürich, CH-8092 Zürich, Switzerland and with the Chair on Non-Invasive Brain-Machine Interface, Center for Neuroprosthetics, School of Engineering, Ecole Polytechnique Fédérale de Lausanne, CH1015 Lausanne, Switzerland, mgublerestudent. ethz . ch little attention paid to developing ways to control remaining paralyzed limbs [2]. If a peripheral reinnervation surgery is not feasible, Functional Electrical Stimulation (FES) is the best alternative to contract paralyzed or paretic muscles [4], [5]. In FES, electrical currents generate an artificial action potential by depolarizing intact peripheral motor nerves that innervate the targeted muscle and cause a muscle contraction. Thus, FES devices can be used to substitute lost neurological functions by bypassing damaged neurons between the central nervous system and the upper extremities.

Currently, FES alone can be used to control simplified (mostly palmar) grasping tasks. However, these tasks cannot yet be naturally executed. Typically, one finger (either the index, middle or ring finger) responds the strongest to the stimulation, thus leading the grasping movement and consequently generating higher local forces at that finger as compared to the other fingers.

Here we describe the development of a passive hand orthosis which aims to support and synchronize the movement of the fingers stimulated by FES for patients with upper extremity palsy. The goals of this work are to (i) enable or improve everyday grasping for disabled people, (ii) to make grasping more ergonomic and natural compared to the existing solutions and (iii) to allow for future control of the orthosis by a brain-computer interface (BCI) [7].

\section{METHODS}

We begin this section by first motivating the need for an FES-driven orthosis, and then extract requirements for the design of such a device. We then go on to describe the chosen design, and finally measure and analyze the distribution of forces provided by the orthosis when operating on everyday objects.

\section{A. Motivation}

The anatomy of the human hand is extremely complex due to the fact that it has to cover an enormous range of applications; such as grasping objects with forceful or precision grasps, for gestural communication, and for the recording of sensory information. In the context of grasping tasks, the hand and the fingers follow a specific pattern to apply force or opposition on an object, with different patterns making up different types of grasps. This work specifically focuses on one of the most widely-utilized grasps of the hand - the palmar grasp [5]. The palmar grasp (also referred to as the power grasp or simply, grasp) is one of the most important types of grasps, and is utilized when grasping larger objects or in situations which require a strong and 
(a)

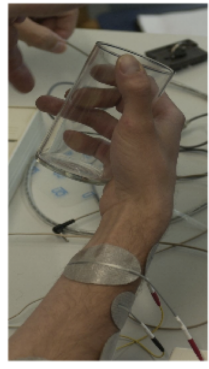

(b)

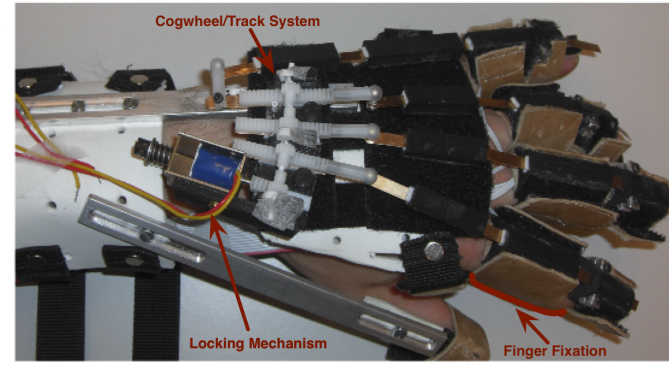

(c)

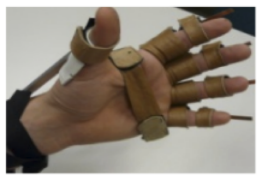

(d)

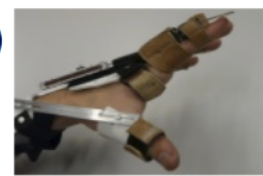

Fig. 1. (a) Unnatural grasping pattern when grasping with the FES. Here, the middle finger provides the strongest force (white finger tip due to lower blood perfusion), while the ring finger has no force contribution. (b) Picture of the orthosis mounted on the backhand and the distal forearm. Sheaths attached with Velcro guide the tendon-like bendable strips from the finger to the synchronization mechanism on the back of the hand, (c) allowing the palm and fingertips to remain free. (d) Additionally the thumb is stabilized.

stable grasp. This grasp is characterized by the four fingers and thumb closing homogeneously around an object, and is quite versatile, allowing for different shaped objects to be firmly grasped, with the greatest forces applied by the index, middle and ring fingers. Thus, this grasp can be characterized in an engineering-sense by synchronizing the four medial finger movements, while stabilizing the thumb.

In a preliminary experiment, the FES surface-electrodes were mounted on the forearm to stimulate finger flexion and extensions, before our passive orthosis was introduced. In these experiments, we observed a well-known problem with FES, in which a single finger (e.g. the middle finger) tends to unnaturally dominate the grasp, as depicted in Figure 1.a.

While FES remains as the best alternative to contract paralyzed or paretic muscles, these unnatural grasping patterns could pose serious safety concerns when grasping large or fragile objects, and are unacceptable for gestures. Thus, this work focuses on overcoming these issues by introducing a supportive orthosis into the FES framework, which synchronizes finger movements and, consequently, distributes the forces applied by each finger. Considering that finger forces are known to change during multi-finger forceproduction tasks [1].

\section{B. Design Requirements}

Throughout the development of the orthosis, many important design considerations have been made, and each can be placed into one of four categories: Aim for Natural Movement, Design for Daily Living, Towards a Brain-Controlled Neuroprosthetic, and Safety.

To achieve Natural Movements while under FES, such an orthosis must be able to synchronize the movement of the four medial fingers and stabilizing the thumb during FES, while consequently equally redistributing the force applied by each finger all meanwhile still allowing for versatile grasps of objects of different shapes and sizes. Furthermore, considerations need to be made in the control of the FES to prevent muscle fatigue from overstimulation.

In the context of Design for Daily Living, such an orthosis must leave the palm free such that a user can easily grab objects allowing for the possibility of tactile or proprioceptive feedback. In addition, the mechanical design and construction of the orthosis must be ergonomic and easily adaptable to different hand sizes, lightweight, robust, inexpensive and available to a wide range of potential users. Finally, a mechanical locking device must be provided to limit the electrical stimulation incurred during long duration grasps, thus reducing muscular fatigue.

Since a distinct goal of this work is to eventually control our orthosis noninvasively with brain signals, we introduce a few considerations that must be made in pursuit of a BrainControlled Neuroprosthetic. In particular, BCIs are capable of distinguishing only a small number of output classes. Since a healthy subject can control a remarkable 24 degrees of freedom (DOF) in the hand, along with an impressive normal range of motion (ROM), the possible DOF and ROM must be limited to enable the possibility of control by a BCI.

Lastly, in the context of Safety, the design must be passive, that is, the fingers should not be mechanically actuated through the use of any active components like motors, but instead by using only the muscular actuation from FES. This requirement offers additional benefits, in particular, by helping to satisfy another requirement - that the orthosis is backdrivable. This means, that since there are no actuators, the orthosis remains flexible and reversible, and can still be opened even in the midst of a grasp.

\section{Components and Design}

Biologically-inspired in its design, the passive orthosis features four bendable strips fashioned from a metallic alloy boasting a full spring-like restoring force, which mimic tendons in their placement, that are mounted unilaterally on the back of each of the four medial fingers, and which serve to apply the forces necessary to operate each individual finger. Those tendon-like bendable strips run above the hand and interconnect over the wrist to synchronize the medial finger movements for a palmar grasp. This is similar to the extensor digitorum communis which diverges under the dorsal carpal ligament into the four tendons on the back of the hand, and inserts into the middle and distal phalanges. Additionally, the thumb is stabilized (see Figure 1.d) and a locking mechanism has been introduced to prevent the need for long-term stimulation during extended grasps. We discuss each of these design components further in the following subsections. 
Individual Fingers: The orthosis makes use of the anatomical center of rotation of each joint and induces flexion and extension moments by pushing and pulling the spring-like metallic bendable strips at a certain lever arm above the joint. In contrast to biological tendons, which can only pull, the semi-rigid strips are able to both pull and push, and can therefore be mounted on only one side of the hand, enabling a free palm (see Figure 1.c). The tendon-like bendable strips are fixed to an ergonomic finger fixation, depicted in Figure 1.b, which is tightly fastened around the middle phalanx of each finger by adaptable leather straps, ensuring long-term comfort, normal blood perfusion and palmar-side tactile feedback. Each bendable strip continues proximally down the finger and is guided through an additional finger fixation that is mounted above the proximal phalanx. These guiding elements are embedded within Teflon sheaths to minimize friction, allowing the strip to exert a pushing force and ensuring that the middle phalanx flexes naturally.

Synchronization: Each of the strips continues down the wrist, where they are mechanically synchronized at a common axis, such that each finger moves together simultaneously. This is achieved through the use of a cogwheel/track system, depicted in Figure 1.b, which operates across all four medial fingers and acts longitudinally on the tendon-like strips to supply a common translation. Since this translation acts on all four medial fingers, the force is shared among all four fingers. And since this sharing is performed on the proximal part rather than on the distal part of the hand, i.e. directly over the held object, a greater variety of objects can be grasped within a far more versatile grasp.

Locking Mechanism: To reduce muscular fatigue caused by long-term stimulation during extended grasps, one aim in the design of the orthosis was to enable the user to hold a grasping pattern without the need for any further stimulation. This was achieved by employing a locking mechanism, which served to lock the position of the cogwheel/track system using the piston of a bi-stable solenoid. Unlock and lock status was toggled by flipping the voltage polarity applied to the solenoid, with its position held magnetically. The coupling of the cogwheel/track system with the solenoid provided a force induced by the lock that was strong enough to allow a user to hold everyday objects, yet it was limited enough to ensure the safety of the user. That is, it guaranteed backdrivability of the system, or rather, it ensured that the hand could be opened at any time if required.

\section{Analysis of Performance}

The improvement in quality of the grasping patterns is quantified in terms of force by examining three configurations alongside of one another; (i) the new orthosis coupled with FES (condition $c O R T$ ), (ii) FES alone (condition $c F E S$ ), and (iii) natural grasping without any FES or orthosis (condition $c N A T)$. A group of healthy subjects tested each configuration on a subset of different objects from daily life; a big cylinder (bottle-like), a small cylinder (glass-like), a spherical object (orange-like), a pen and a book. The pressure between

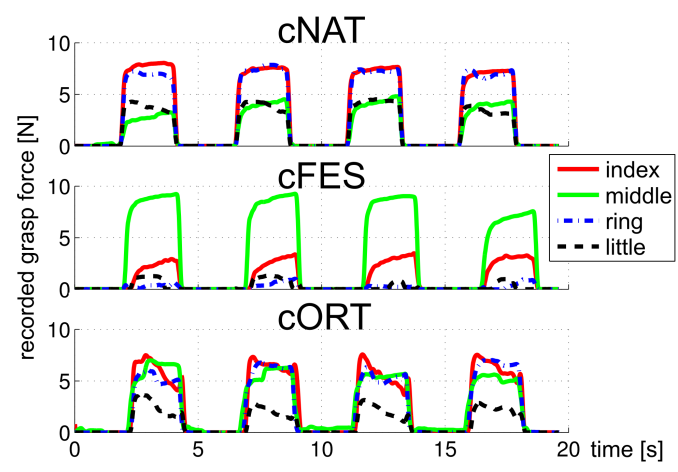

Fig. 2. Forces measured at the fingertips for several grasps by a healthy subject on a cylinder for one healthy subject. $c N A T=$ Natural grasp, $c F E S=F E S-$ stimulated grasp (without orthosis), $c O R T=F E S-$ stimulated grasp with orthosis.

each finger and the object was recorded with linear pressure sensors (FSR-400, IEE, Luxembourg, $8 \mathrm{~mm}$ diameter) over the joint capsules of the fingertips, exclusively measuring force in the normal direction. The force recordings were acquired at a sampling rate of $100 \mathrm{~Hz}$ via a data acquisition device (NI-USB-6008, National Instruments, USA). The FES stimulation was controlled by a microcomputer-controlled stimulator (MotionStim 8, Krauth \& Timmermann, Germany) which performed stimulation at $20 \mathrm{~Hz}$, with a $300 \mu$ s pulse width, and currents between $3 \mathrm{~mA}$ and $16 \mathrm{~mA}$ (dependent on the subject). Multiuse electrodes (Krauth \& Timmermann, Germany) stimulated the fingers extensor muscle (musculus extensor digitorum comunis) for the extension of the four medial fingers. Hand closing was achieved by stimulating the fingers flexor muscle (musculus flexor digitorum superficialis).

Five repetitive hand opening and closing exercises were performed without the use of the locking mechanism, each for a duration of two seconds on every grasped object for each configuration (FES-alone, natural grasping, FES+orthosis), by healthy subjects.

\section{RESULTS}

For all five subjects, the grasps performed with the orthosis (cORT) moved more synchronously and were qualitatively more natural than grasps initiated by the FES alone ( $c F E S$ ). Across all subjects, it was observed that, with the orthosis, no single finger dominated the grasps - instead, all fingers moved simultaneously. Furthermore, each subject reported that they felt more comfortable grasping with the orthosis than with FES alone.

A trial performed in each configuration on a single subject is depicted in Figure 2. For $c N A T$, the force-contributing fingers (index and ring finger) show similar and synchronized amplitudes, while the middle and little finger produce slightly smaller forces. In the $c F E S$ configuration, marked differences appear- a dominant middle finger dominates the grasp, exerting a far greater force on the object than the other medial fingers, which are not co-actuated at all. However, when the orthosis is introduced and coupled with FES in the $C O R T$ configuration, force amplitudes are better 


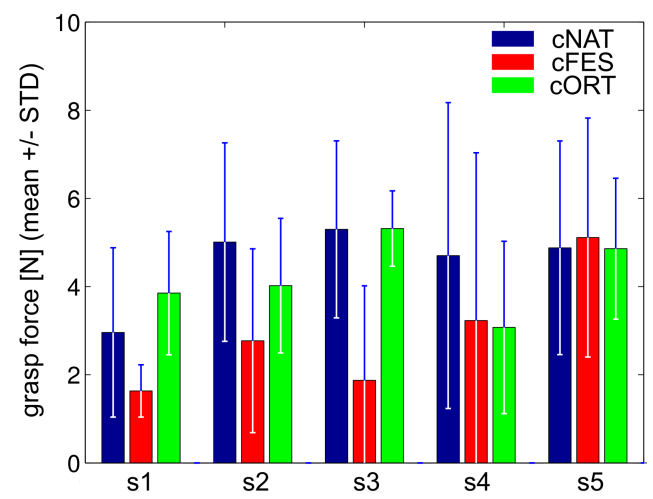

Fig. 3. Averaged forces $( \pm$ STD) of five repetitive grasps of the object big cylinder for each subject and condition: natural grasp ( $c N A T)$, grasp with FES (cFES) and grasp with orthosis and FES (cORT).

synchronized, meanwhile providing a grasping pattern that more closely resembles a natural grasp.

These improvements were quantified by comparing the recorded forces for the three configurations. Grand averages revealed for cNAT forces of $4.6 \pm 2.4 \mathrm{~N}$, for cFES reduced forces of $2.9 \pm 2.6 \mathrm{~N}$ and for cORT recovered ones of $4.2 \pm 1.6 \mathrm{~N}$. Due to the physiological differences affecting grasp strength between subjects, differences in sensor placement on each subject, as well as differing placements of the grasped object within the hand, we considered only mean values over time and repetitions, and we depict these measurements per subject in Figure 3. Due to these differences, we are more interested in the comparison of the results per individual subject. Therefore, the higher mean value together with the lower STD of the finger pressures seen in Figure 3 while wearing the orthosis clearly indicates that the force distribution in $c O R T$ was more homogeneous than in $c F E S$.

\section{DISCUSSION}

The main aim of this work was to synchronize the fingers during grasping in an effort to overcome the unnatural grasping pattern caused by FES stimulation alone. This synchronization was successfully achieved through the development of a simple, passive hand orthosis that homogenized the finger movements, allowing for a more natural grasp. These improvements were not only observed qualitatively, but quantitatively through force recordings made on each of the fingertips.

The quantitative results provided by the force sensors were expected to give a clearer trend towards homogenization, but several factors influenced the recordings: First, the pressure sensors placed on the fingers had to make contact with the object perpendicular to the object's surface. Secondly, not every grasp made in the $c N A T$ configuration was made using the same strength. In $c F E S$ and $c O R T$ the same current is applied by the FES, but the position of the finger (and therefore the sensor) on the object varied with each grasp. This variation is observed among all subjects and within each configuration. Last but not least, not all the subjects could withstand the same amount of FES current, so the output force was not same for all subjects.
A possible contributor to some of these issues might be backlash. For example, when holding an object, the orthosis might be in a locked position but the hand is not forced rigidly in place. Instead, a small amount of movement might be possible between states, which are referred to as backlash. This backlash was induced (i) by the attachment of the orthosis to soft tissue, (ii) by the tendon-like strips which are not always guided perfectly, and (iii) by the flexible mounting with Velcro. A personalized exoskeleton could reduce this backlash.

\section{CONCLUSIONS AND FUTURE WORK}

In summary, a passive orthosis for the use with FES was developed which offered a considerable improvement over FES alone. Such an orthosis in combination with FES could allow disabled people to regain the ability to perform grasps in their daily life. In fact, each healthy participant reported that they felt more confident in grasping with rather than without this orthosis. This remains to be seen, however, with patients.

Since this work was undertaken in an effort to develop a brain-controlled prosthetic, further work involves coupling our FES orthosis with a BCI [7]. However, a well-known problem with this coupling is the electrical and neurological interference introduced into the EEG by the FES stimulation. Thus, the lock developed for our orthosis not only serves to allow for long-term grasps by locking the fingers and toggling off stimulation to prevent muscular fatigue- it also allows for the possibility of control via BCI. This is because the BCI command can be detected while no FES stimulation is provided, and thus, no interference triggered by the FES on the EEG should influence the BCI. Further experiments to control the FES grasping patterns with a BCI must still be undertaken, although preliminary results have already been produced and are presented at this conference [6].

\section{REFERENCES}

[1] F. Danion, G. Schöner, M. L. Latash, S. Li, J. P. Scholz and V. M. Zatsiorsky, "A mode hypothesis for finger interaction during multifinger force-production tasks", Biol Cybern., vol. 88, no. 2, pp. 91-98, 2003.

[2] T. A. Kuiken, G. A.Dumanian, R. D. Lipschutz, L. A. Miller, and K. A. Stubblefield, "The use of targeted muscle reinnervation for improved myoelectric prosthesis control in a bilateral shoulder disarticulation amputee," Prosthet Orthot Int., vol. 28, no. 3, pp. 245-253, 2004.

[3] G. Müller-Putz, R. Scherer, G. Pfurtscheller, and R. Rupp, "Braincomputer interfaces for control of neuroprostheses: From synchronous to asynchronous mode of operation," Biomed. Technik, vol. 51, pp. 5763, 2006.

[4] P. H. Peckham, M. W. Keith, K. L. Kilgore, J. H. Grill, K. S. Wuolle, G. B. Thrope, P. Gorman, J. Hobby, M. J. Mulcahey, S. Carroll, V. R. Hentz, A. Wiegner, "Efficacy of an implanted neuroprosthesis for restoring hand grasp in tetraplegia: a multicenter study," Arch Phys Med Rehabil., vol. 82, no. 10, pp. 1380-1388, 2001.

[5] R. Rupp and H. J. Gerner, "Neuroprosthetics of the upper extremityclinical application in spinal cord injury and future perspectives," Biomed. Technik, vol. 49, pp. 93-98, 2004.

[6] M. Tavella, R. Leeb, R. Rupp and JdR. Millán, “Towards Natural NonInvasive Hand Neuroprostheses for Daily Living," Proc. IEEE-EMBC conference, accepted 2010.

[7] J. R. Wolpaw, N. Birbaumer, D. J. McFarland, G. Pfurtscheller, and T. M. Vaughan, "Brain-computer interfaces for communication and control." Clin Neurophysiol., vol. 113, no. 6, pp. 767-791, 2002. 